\title{
DESCRIPCIONES Y CLASIFICACIONES EN DERECHO
}

ROBERTO J. VERNENGO

UnIVERSIDAD DE BUENOS AIRES

CONICET

Se ha convertido en tradicional la distinción entre una consideración descriptiva y un enfoque normativo cuando hablamos de derecho. Así, en Kelsen. Para este autor, el derecho está constituido por normas, enunciados lingüísticos con función principal prescriptiva: se trata de oraciones usadas para provocar conductas en los receptores de los mensajes. Este conjunto de enunciados, las normas jurídicas, configuraría, en algunas culturas, el objeto de un conocimiento con pretensión científica. La ciencia del derecho así considerada se ocuparía de las normas jurídicas, y sus enunciados, aunque se refieren a normas prescriptivas, sólo pretenderian describir dichos objetos. La ciencia del derecho sería así una ciencia descriptiva del derecho positivo (i.e., conjunto de normas prescriptivas) de que se ocupe. ${ }^{1}$ Esta distinción se remonta a la distinción, corriente en el pensamiento filosófico de nuestro siglo, entre enunciados descriptivos y enunciados prescriptivos o valorativos. Los primeros son susceptibles de confirmación o falsación por alguna experiencia empirica, y su verdad es considerada, entonces, como una verdad de hecho, fáctica. Los enunciados prescriptivos ( $\mathrm{y}$ también los valorativos que suelen ser reducidos a enunciados normativos), en cambio, en su función prescriptiva, no admiten ser confirmados o falseados por experiencias empíricas y, por ende, no tienen un valor de verdad similar al de los enunciados descriptivos. Más aún, por lo general se sostiene que los enunciados normativos prescriptivos carecen radicalmente de valor de verdad.

1 Cfr. H. Kelsen, Reine Rechtslehre, 2a. ed., Viena, 1960, p. 16, donde se afirma que "die Rechtswissenschaft kann das Recht nur beschreiben", tesis que Kelsen subraya. La misma se encuentra ya en textos anteriores. Por ejemplo, en la General Theory of Law and State, de 1945 , se declara que "like any other empirical science, normative jurisprudence describes its particular object", $y$ lo hace de suerte que "an analytical description of positive law as a system of valid norms is, however, no less empirical than natural science restricted to material given by experience". En ese sentido: "the theory of positive law is parallel to the empirical science of nature" (p. 163). 
La ciencia del derecho - título que se suele atribuir a la dogmática jurídica elaborada académicamente desde mediados del siglo pasado- sería una ciencia descriptiva, a saber: descriptiva de las normas que constituyen los derechos positivos. Cuál sea el estatuto teórico de las ciencias descriptivas es asunto discutible, cuando encaramos el tipo de discurso que las disciplinas jurídicas despliegan. Por de pronto, la ciencia del derecho poco se parece a disciplinas tradicionalmente vistas como descriptivas, como la geografía o la botánica clásicas, pues éstas, aunque elaboren su material de forma teóricamente rudimentaria, pretenden que sus enunciados descriptivos sean objetivamente verdaderos. Esta característica ha sido muchas veces negada a las ciencias jurídicas, sea que rotundamente se les rehúse enteramente carácter de conocimiento teórico, sea que se las considere incapaces de lograr conocimientos objetivos. Suele afirmarse que, cuando a la jurisprudencia nos referimos, se trataría más bien de un discurso puramente ideológico construido con fines retóricos -tesis que en las últimas décadas fue reiterada hasta el cansancio, sobre todo en el pensamiento de inspiración marxista. $Y$ dado que sus enunciados tienen, en una interpretación posible, una modalidad normativa, las supuestas descripciones de las ciencias jurídicas no constituirian sino normas encubiertas: la ciencia del derecho, en rigor, sería parte del derecho positivo mismo, sea en cuanto fuente del mismo, sea en cuanto normas prescriptivas subrepticiamente introducidas en los órdenes jurídicos so capa de su prestigio científico.

Sucede, por de pronto, que la característica descriptiva de los enunciados de la ciencia jurídica no es cosa evidente de suyo. La tesis kelseniana puede ser vista como un subterfugio para salvar la cientificidad de la jurisprudencia y renegar de su carácter ideológico, pero en los textos de su autor no encontramos un análisis suficiente de la noción de ciencia descriptiva. Más aún, la noción de enunciado descriptivo, en contraposición a enunciados normativos o valorativos, dista de haber sido analizada satisfactoriamente. Justamente, J. Esquivel destacó estas dificultades: "La distinción entre descriptivo y evaluativo [...] se ha confundido frecuentemente con otras distinciones, como descriptivo/prescriptivo, ser/deber-ser, fáctico/normativo, científico/no-científico, usándose todas ellas de manera intercambiable. Estas distinciones son diversas y separadamente interesantes."2 En especial, Esquivel destaca cómo en la confusión entre descripción y prescripción, el criterio de la atribución de verdad o falsedad a los respectivos enunciados constituiría un criterio suficiente de la distinción, al funcionar como una partición. "Las normas o imperativos no pueden ser

2 J. Esquivel, "Juicios de valor, positivismo jurídico y relativismo moral", en Crítica, vol. XIII, no. 37, p. 4 ss. Las referencias al pensamiento de Esquivel que hago en estas páginas deben buscarse, salvo indicación en contrario, en ese ensayo. 
ni verdaderos ni falsos; no tienen una relación de correspondencia con el mundo, ni nos dicen cómo es, sino que, por el contrario, es el mundo el que debe ajustarse a ellos", características todas que, en cambio, se suelen atribuir a los enunciados descriptivos.

Pero la noción de descripción no siempre es asimilable a la de enunciado de hecho. "Normalmente, al describir un objeto, una persona o una situación, lo que se hace es proporcionar una serie de características que dan una imagen del mismo. Es importante recalcar -indica Esquivel- algunos de los aspectos que podemos señalar en esta operación y que ayudan a tener una correcta comprensión de lo que se entiende por 'describir' en el uso cotidiano. Lo que comúnmente decimos de una descripción es que es detallada, apropiada, grotesca, etc. Sus méritos, cuando pensamos por caso en la de un novelista, no son siempre el ser exacta o detallada, sino el ser vívida, conmovedora, etc. Como el objetivo de una descripción es ser verdadera, sería muy extraño decir que la presente descripción es falsa y más bien decimos que es engañosa, inadecuada o superficial. Una falsa descripción no es realmente descripción de nada." O sea, que si el criterio de distinción reposa en la atribución de verdad o falsedad (i.e., no verdad), resulta que las descripciones, en sus usos corrientes, no pueden ser vistas como falsas: una descripción falsa no constituye descripción alguna. Pero, para esta forma de ver el asunto, si una descripción no puede ser falsa, en rigor tampoco puede ser verdadera y, por ende, escapa al criterio de distinción adoptado para diferenciar enunciados normativos de enunciados descriptivos. De estar constituida la ciencia del derecho por enunciados descriptivos de normas, tendríamos que los mismos carecerían de valor de verdad, $y$, por ende, quizás correspondiera atribuir razón a los que ven en los textos teóricos de los juristas nada más que un discurso normativo encubierto.

Por añadidura, como también apunta Esquivel, "las descripciones no están siempre en la forma gramatical del indicativo, ni son equivalentes a enunciados de hecho. Así, por ejemplo, 'son las tres de la tarde' es claramente un enunciado fáctico, pero difícilmente puede pasar por ser una descripción; en todo caso sería parte de una descripción. Es también relevante notar que las descripciones pueden ser emotivas, por ejemplo, conmovedoras, y de ninguna manera excluyen el uso de nociones morales. Más todavía, cualquier descripción de un ser humano, en particular de su personalidad y carácter, es necesariamente incompleta si no se incluye una referencia a sus cualidades morales." $Y$ concluye Esquivel, destacando dos resultados "interesantes": el primero, que la característica de descriptivo atribuida a un enunciado "no clasifica gramaticalmente una clase de oraciones o palabras, sino un modo de usar oraciones y palabras". El segundo, que el uso que se hace de tal característica, el ser descriptivo un enunciado, "tal como aparece 
en los filósofos y, en especial, en los filósofos de la moral y del derecho, es un uso técnico que se aparta del uso común".

Si atendemos a la segunda conclusión, corresponderá dilucidar el significado técnico, en tal o cual autor, del término "descripción" o "enunciado descriptivo" y, a la postre, el de "ciencia descriptiva". Por de pronto, conforme al primer resultado apuntado, la distinción no se cumpliría en el plano sintáctico (donde gramatical y lógicamente cabe convenir características distintivas entre enunciados descriptivos y enunciados prescriptivos) ni en el semántico, pues la propiedad semántica por excelencia, la de verdad, no tiene papel alguno respecto de las normas y, como Esquivel afirma, es de dudosa aplicación a las descripciones. De ahí que la caracterización de la ciencia jurídica como una disciplina descriptiva, en Kelsen, sea insatisfactoria: "En el plano lógico-lingüístico Kelsen es particularmente pobre en el señalamiento de la sintaxis y la semántica que subyacen a su modo de ciencia jurídica." ${ }^{3}$ La distinción entre descripción y prescripción tiene más bien un carácter pragmático: se trata de modos diversos de usar oraciones y palabras. Pero sucede entonces que la distinción, que tenía un carácter suficiente si se tomaba como criterio de la partición el contar con valor de verdad o no — esto es, una propiedad típicamente semántica-, queda desdibujada, pues se reduce a lo sumo a la distinción entre oraciones con referencia y oraciones no referenciales. Considerar que la distinción entre descripciones y prescripciones es de índole pragmática lleva a pensar que no estamos frente a una distinción suficiente, pues, en ese plano, no cabe sostener que tengamos una partición que haga a los usos prescriptivos del lenguaje excluyentes de los usos descriptivos. La teoria del lenguaje y del derecho, justamente, se ha debatido frente a la circunstancia de que no cabe establecer usos prescriptivos rígidos y absolutos de las expresiones lingüísticas, como lo abona la interpretación corriente del conocimiento teórico del derecho como una forma más de discurso ideológico prescriptivo.

Esquivel, luego de recordar otras dificultades que surgen de la distinción entre enunciados descriptivos y enunciados prescriptivos, apunta que "en muchos autores la distinción entre descriptivo, prescriptivo y evaluativo tuvo propósitos muy sanos, como el de disipar la confusión entre leyes naturales y leyes jurídicas, así como el de posibilitar una posición neutral frente a los debates ideológicos, sobre todo en el terreno de las ciencias sociales y la moral". El peligro que esta tajante distinción y el ideal de

3 J. Esquivel, Kelsen y Ross: formalismo y realismo en la teoría del derecho, UNAM, 1980, p. 174. Cabe señalar, ateniéndose a esta exposición de Esquivel, que también en A. Ross encontramos la tesis del carácter descriptivo de la ciencia del derecho, sólo que ahora se trata de la descripción de "ciertas realidades sociales", a las cuales se refieren en "última instancia [... ] las proposiciones de la ciencia del derecho" (ibid., p. 117). 
neutralidad suscitaron fue incurrir en relativismo moral, posición con la cual Esquivel no simpatizaba. En concreto, refiriéndose a la posición de Kelsen, Esquivel señala que si para la teoría pura "la ciencia del derecho tiene por objeto las normas jurídicas y éstas ni son hechos, ni pueden reducirse a hechos", resulta que la descripción jurídica que Kelsen propone no es una descripción fáctica ni empírica y, por ende, los enunciados descriptivos de la ciencia jurídica, los Rechtssätze, si son verdaderos o falsos, no lo son al modo de los enunciados empíricos. De ahí -prosigue Esquivel- que "la idea que parece tener Kelsen de un científico del derecho es la de alguien que va a describir el derecho con la misma actitud con que se describen los fenómenos naturales. Pero esto no es así; los actos jurídicos, como él mismo lo reconoce, no son perceptibles a la manera como se perciben las propiedades naturales de un objeto, como el color, la dureza, el peso. Por ser actos racionales tienen que ser comprendidos, no como un trozo de naturaleza [... ] determinado por leyes causales sino conociendo su sentido, en especial, el sentido objetivo que les dan las normas jurídicas." 4

En definitiva, la ciencia jurídica descriptiva que propone Kelsen no constituye una ciencia empírica y no considera a su objeto inmediato - las normas positivas- fenómenos empíricos, sino sentidos ideales. ${ }^{5}$ Cabría pensar, pues, que la ciencia del derecho, que ciertamente no se interesa por los aspectos puramente lingüísticos superficiales de los enunciados normativos, se configuraría como una suerte de análisis semántico en el que, a partir de los enunciados dados, se trata de establecer la existencia de sus referentes ideales. Pero esto lleva a una ontología innecesaria, que parece contraria a los desarrollos efectivos que Kelsen ha efectuado en la teoría del derecho y que no corresponde a la labor que los juristas hacen.

Propondré, en una primera aproximación, que el carácter descriptivo que se atribuye a la ciencia del derecho positivo consiste en su actividad clasificatoria, una forma de teoria de nivel quizás elemental, pero propia de muchas otras ciencias históricamente prestigiosas. La actividad básica de los juristas consiste en clasificar el material normativo. Tal cosa, sin embargo, no es del todo clara. Textos clásicos de la teoría general del derecho sostienen que la ciencia dogmática cumple una actividad descriptiva y sistematizadora del material normativo, esto es, del derecho positivo de que se trate. Por de pronto, fuera de los reparos conceptuales que Esquivel ha formulado, es notorio que la jurisprudencia dogmática que, según se dice, describe el derecho objeto de su estudio, no lo hace con función explicativa,

4 Cfr. op. cit., nota 1, p. 15 y 17.

5 Esta idea fue expresamente adoptada por H. Kelsen en la Allgemeine Theorie der Normen, p. 22, inter alia. Cfr. R.J. Vernengo, Truth and Validity in Law and Morals, p. 731 ss., en Staatsrecht in Theorie und Praxis, Viena, 1991. 
ni se reduce tampoco a una descripción fenomenológica de ciertos datos. Por cierto que los criterios de identificación de los datos considerados, por de pronto, jurídicos y, además, parte de cierto derecho positivo, es asunto que la jurisprudencia dogmática asume como un presupuesto rara vez hecho explícito. Pero suponiendo que se trate de cierta clase de enunciados normativos, es claro que su clasificación puede efectuarse desde distintos puntos de vista y tomando en cuenta diversos elementos componentes. Para una clasificación puramente lógica, las modalidades normativas utilizadas -prohibiciones, deberes y facultades - constituyen una partición suficiente, pero cuál sea la lógica modal deóntica a la que se recurre puede ocasionar divergencias en la taxonomía: así se encuentran en la literatura dogmática referencias a derechos-deberes, como sucede con algunos de los derechos humanos consagrados por documentos internacionales y muchas constituciones. Piénsese en el derecho al nombre o en el derecho a la personalidad jurídica. Tal caracterización es, en principio, contradictoria y no define un conjunto interesante de datos (normas), toda vez que suscita consecuencias triviales. No se trataría de una taxonomía aceptable. Parece, pues, que una labor importante de la ciencia del derecho y de su teoría general presupuesta es establecer criterios de clasificación teóricamente adecuados.

Pero estas clasificaciones a partir de características lógicas - como otras fundadas en supuestas clasificaciones de las formas lógicas o gramaticalescarece de alcance sustantivo. Quedamos en el terreno enteramente vacío de la pura lógica. Si bien queda garantizada la coherencia del discurso lógico, desde un punto de vista teórico material tales clasificaciones no avanzan ni un ápice en la riqueza teórica de la ciencia del derecho. Por ello, es frecuente tachar a tales esquemas, cuando son tomados como criterios exclusivos de clasificación, de formalistas. Y si bien la categorización puramente formal de los datos es condición necesaria de todo conocimiento científico objetivamente válido, no lo es para alcanzar un conocimiento materialmente satisfactorio que signifique incrementar y elaborar la información en bruto que se da con los datos de los que se parte.

Leibniz habría sido quien subrayó, aunque en son de crítica, que la labor teórica de los juristas consiste en elaborar complicadas e inútiles clasificaciones. Las mismas han sido tradicionalmente resguardadas, de suerte que el jurista actual tiende a manejarse con clasificaciones y taxonomías que quizás se remontan al escaso o nulo interés teórico de algún pontífice romano, como aquellas de ius in rem, in personam, que más que pautas clasificatorias remiten a ritos mágicos olvidados. Aquel perspicaz filósofo señaló también otra característica del afán clasificador de los juristas: suelen satisfacerse, como se dijo arriba, con clasificaciones puramente formales, aunque, formalmente hablando, la lógica o las lógicas a que recurren pueden ser harto 
endebles. De ahí resulta que el discurso de la ciencia del derecho aparezca por lo general con una pretensión normativa excesiva: las normas positivas traídas a cuento en alguna clasificación propuesta, parecieran simples ejemplos significativos de cómo las actividades legislativas sociales han de ajustarse a los marcos conceptuales de los teóricos del derecho. Las opiniones de los juristas, en consecuencia, tendrían tanta o más autoridad que los pronunciamientos del legislador.

Para designar la actividad clasificadora de los juristas diré que se trata de categorizar los datos, esto es, incorporarlos en algún casillero clasificador conceptualmente concebido. El jurista encara sus datos desde los casilleros de alguna teoría, toda vez que una clasificación constituye, en un nivel elemental, una especie de teoría ${ }^{6}$ Cabe apuntar que, como Hempel señala, debemos contar en todo caso con especificación de las condiciones suficientes y necesarias para la inclusión de las entidades clasificadas en el casillero taxonómico correspondiente. Ello puede efectuarse extensionalmente - como aquella vieja clasificación de las obligaciones-, o intensionalmente, mediante conceptos que definan el conjunto de propiedades necesarias para atribuir pertenencia a la clase. El nivel teórico de las ciencias da a estas pautas distinto alcance: desde ciencias ingenuamente descriptivas, hasta taxonomías erigidas a partir de complejos conceptos teóricos. Cada rango jerárquico en la clasificación debe estar así definido de alguna suerte. Ello se logra mediante un conjunto de conceptos clasificatorios. Tratándose de ciencias con referencia empírica, la terminología de la misma tiene como función permitir una descripción adecuada de los objetos que se investigan. Estas descripciones iniciales constituyen, por lo común, generalizaciones empíricas, esto es, enunciados generales con predicados observables. Así, en derecho, quizás podrían haberse distinguido los delitos por el tipo de consecuencias verificables que exhibían: en algunos casos daban lugar a la pena capital, en otros a represalias y venganzas, etc. Pero esta etapa inicial es de escasa importancia en el campo de la ciencia jurídica, donde pronto entran a desempeñar un papel importante criterios clasificatorios y sistemáticos derivados de criterios no directamente observables, sino mas bien atribuibles en mérito a construcciones conceptuales cada vez más complejas. Por ejemplo, el contar con ciudadanía en Roma o ser extranjero. Claro que, en este respecto, las ciencias del derecho, desde su inicio en Roma, difieren de las clasificaciones naturales de disciplinas como la biología o la zoología antiguas, pues no pretenden tener valor explicativo causal. Se trata de la introducción de conceptos teóricos sin valor etiológico.

6 C.G. Hempel, "Fundamentals of Taxonomy", en Aspects of Scientific Explanation and Other Essays in the Philosophy of Science, Londres, 1965, cap. III, art. 6. 
A los romanos, por ejemplo, les interesaba mucho concebir adecuadamente los diferentes tipos de obligaciones, dado que los procedimientos a que cabía recurrir para hacerlas efectivas - procedimientos derivados, como se sabe, de prácticas mágicas primitivas-diferían grandemente. De ahí que se introdujeran clasificaciones con pretensión de objetividad, mediante el recurso a definiciones operacionales con las que cualquier interesado pudiera alcanzar resultados idénticos con independencia de su posición subjetiva. Por lo tanto, desde temprano, en la jurisprudencia romana aparecen clasificaciones artificiales. Hempel señala que las clasificaciones consideradas naturales resultan de criterios de pertenencia a un casillero clasificatorio que están asociados a muchas otras características concomitantes que, por su carácter heterogéneo, las hacen teóricamente poco fecundas. Así, en derecho, muchas clasificaciones de los datos jurídicos que implican contenidos valorativos -por ejemplo, aspectos morales o económicos-, fueron desdeñados a favor de clasificaciones más artificiales en que tales conjuntos de características eran depurados. Distinguir responsabilidades por dolo o por culpa supone recurrir a conceptos mucho más alambicados que no pueden ser definidos ostensivamente. Clasificar los delitos por los supuestos bienes protegidos tiene, en cambio, un alcance teórico inferior, en cuanto no permite generalizaciones interesantes $y$, por ende, ofrece poco campo a predicciones.

En la jurisprudencia, pues, se elaboran clasificaciones a partir de categorías teóricas, pero su rendimiento sistemático no es muchas veces tomado en cuenta. Frente a un dato cualquiera - pongamos por caso, cuál es el nombre que la mujer casada puede o debe exhibir- el jurista sitúa rápidamente la cuestión en campos clasificatorios que integran una partición. Puede que considere, en un cierto derecho positivo, que la mujer casada tiene un nombre determinado obligatorio; por ejemplo, puede estar legalmente obligada a llevar el apellido del marido. Puede que le quede prohibido seguir usando su nombre familiar anterior. En el derecho argentino actual, la legislación dice que la mujer tiene derecho —es decir, está jurídicamente facultada-a mantener su propio apellido, usarlo conjuntamente con el apellido marital, o adoptar el apellido del cónyuge. Se trata, pues, de una facultad disyuntiva. Como su ejercicio requiere de una manifestación de voluntad, el jurista ubica tal facultad en un dominio más amplio: el de actos jurídicos (para distinguirla de los llamados hechos juridicos). Al considerar que la facultad de escoger el nombre de casada es un acto jurídico, el jurista extiende las condiciones y requisitos de los actos jurídicos válidos al tema que intenta pensar correctamente. Pero los actos jurídicos pueden ser bilaterales, como en el caso de un contrato, o pueden ser manifestaciones unilaterales, como en el caso de un testamento. Claramente, al situar la elección del 
nombre en el campo de los actos jurídicos unilaterales, se incorporarán al caso nuevas normas y principios relativos a la validez de tales declaraciones unilaterales, así como a sus efectos frente a terceros, etc. La clasificación así elaborada incorpora el tema a un marco teórico más elaborado: la teoría de los actos civiles y, por ende, permite prever la aplicación de otras normas, quizás no tenidas en cuenta en el planteamiento inicial del asunto.

Estas teorías, como muchas otras que la jurisprudencia romanista mantiene y desarrolla, no son, por su carácter normativo, susceptibles de verificación empírica inmediata, aunque sí cabe descubrir que algunas permiten predicciones suficientes respecto de la aplicación efectiva de otras normas. Que la elección del nombre sea un acto civil unilateral involucra que deben satisfacerse ciertas condiciones, por ejemplo, relativas a la capacidad del sujeto y, eventualmente, a la forma del acto. Pareciera que muchas de estas construcciones taxonómicas de la jurisprudencia romanista tienen origen en ideas filosóficas griegas, en especial, ideas derivadas de la ética estoica, aunque algunas - como Kelsen intentó mostrarlo eruditamente con la noción de culpa y de imputabilidad penal - se remontan a tesis más arcaicas. Sea cual fuere su origen, la ciencia dogmática reciente las utiliza con propósitos teóricos y, básicamente, con la forma de clasificaciones. Entiendo que el llamado carácter descriptivo de la ciencia jurídica, al que me refería en las primeras páginas de este ensayo, corresponde a una primera etapa en esa tarea taxonómica a la cual, muchas veces, se reduce el conocimiento científico del derecho. 